\title{
GOBERNANZA Y ECOLOGÍA POLÍTICA EN LA GESTIÓN DEL REGADÍO MULTIFUNCIONAL. APLICACIÓN AL CORREDOR AGROECOLÓGICO EL HONDO-LOS CARRIZALES (ALICANTE)
}

\section{RESUMEN}

La gestión del regadío multifuncional se viene debatiendo en términos que incorporan tanto la preocupación por su impacto socioeconómico y ambiental, como discusiones acerca de los intereses público-privados que emanan de su promoción. Con ello, las cuestiones sobre quién forma parte de la toma de decisiones y en qué medida participa o ejerce cierto control o influencia sobre el conjunto de actores centran el debate sobre su legitimidad y gobernanza. El presente artículo profundiza en estos aspectos de la mano de un caso de estudio, el corredor agroecológico que conforma el Parque Natural El Hondo y el Parque Natural Agrario Los Carrizales (Elche, Alicante). En base a la realización de entrevistas semi-estructuradas y cuestionarios y con el fin de profundizar en su modelo de gestión y las relaciones de poder entre partes interesadas, este artículo analiza 1) las cuestiones que generan mayor preocupación y debate en el sistema agroecológico El Hondo-Los Carrizales y 2) el nivel de gobernanza en la toma de decisiones que emana de su gestión. Los resultados muestran un reconocimiento social escaso del carácter multifuncional de ambos parques, así como la necesidad de afianzar su gestión de forma integrada y adaptativa para con ello aumentar su resiliencia frente a los impactos del cambio climático y maximizar su potencial agronómico, ambos dos aspectos clave para la supervivencia del sistema El Hondo-Los Carrizales.

a Geógrafa. Investigadora postdoctoral. Instituto Interuniversitario de Geografía, Universidad de Alicante sandra.ricart@ua.es

b Geógrafo. Catedrático de Universidad. Instituto Interuniversitario de Geografía, Universidad de Alicante am.rico@ua.es

Fecha de recepción: 28/2/19. Fecha de aceptación: 28/11/19. 
Palabras clave: gobernanza, ecología política, regadío, corredor agroecológico, El Hondo, Los Carrizales, Alicante

\title{
GOVERNANCE AND POLITICAL ECOLOGY IN THE MANAGEMENT OF MULTIFUNCTIONAL IRRIGATION. APPLICATION TO THE AGROECOLOGICAL CORRIDOR EL HONDO-LOS CARRIZALES (ALICANTE)
}

\begin{abstract}
The management of multifunctional irrigation has been debated both in terms of the concern for its socio-economic and environmental impact, and discussions about the public-private interests that emanate from its promotion. According to this, questions about who takes part of the decision-making processes and to what extent they participate or exert some control or influence on the whole stakeholders focus the debate about its legitimacy and governance. This article delves into these aspects from the analysis of a case study, the agroecological corridor formed by the El Hondo Natural Park and Los Carrizales Agrarian Natural Park (Elche, Alicante). From semi-structured interviews and questionnaires and in order to deepen on its management model and the power relations between key stakeholders, the paper analyses 1) the issues that generate the greatest concern and debate on the El Hondo-Los Carrizales agroecological system and 2) the level of governance in decision-making process. Results show a lack of social recognition towards the multifunctionality of both parks, as well as the need to strengthen its management in an integrated and adaptive way, thereby increasing its resilience to the impacts of climate change and maximizing its agronomic potential, both key aspects for the survival of the agroecological system of El Hondo-Los Carrizales.

Key Words: governance, political ecology, irrigation, agroecological corridor, El Hondo, Los Carrizales, Alicante
\end{abstract}

\section{INTRODUCCIÓN}

El debate en torno al futuro de la agricultura y el frágil equilibrio entre el beneficio socioeconómico (productividad) y el impacto ambiental (sostenibilidad) no es nuevo (Wi1son, 2009; Seufert et al., 2012; Peng et al., 2015). Sin embargo, con los años se ha ido configurando un cierto consenso al respecto, según el cual la agricultura no debe limitar su función a la producción de materias primas para la industria agroalimentaria, al contrario, pues debe incorporar una mayor exigencia en la calidad y seguridad de los alimentos, el bienestar animal y las consideraciones ambientales (Seuneke et al., 2013; Rizzo, 2016). Es lo que se viene denominando la multifuncionalidad de la agricultura, entendida como la dimensión socio-ecológica de la actividad agraria, a saber: que la agricultura se asocie a aspectos como la generación de bienes comunes, la promoción de la identidad local, la generación de empleo y de capacidad de negocio, refuerce los parámetros de la seguridad alimentaria o promueva 
el desarrollo rural (Morgan et al., 2010; Ricart, 2016). Sin embargo, asumir nuevas funciones requiere cambios en la estructura tradicional agraria (Duru et al., 2015), como las innovaciones tecnológicas, una actitud proambiental, nuevas estrategias de mercado o asumir las nuevas demandas del consumidor, etc. Entre todas ellas, una condición destaca por su transversalidad: la provisión de servicios ecosistémicos. Este concepto se introdujo a principios de los años ochenta como una iniciativa conjunta entre economistas y ecologistas por la que se ponía en valor los servicios que genera la naturaleza en beneficio de las actividades productivas, como la agricultura (Gómez-Baggethun et al., 2010).

Los servicios ecosistémicos guardan una estrecha relación con la multifuncionalidad agrícola. Ambos conceptos son antropocéntricos porque se centran en los beneficios sociales que genera la agricultura y porque se concibieron para abordar un objetivo similar: identificar qué tipo de compensaciones se podían establecer entre la actividad agrícola y la protección ambiental (Swinton et al., 2007). Una interesante revisión de la literatura realizada por Huang et al. (2015) sugirió que ambos conceptos, multifuncionalidad y servicios ecosistémicos, se desarrollaron de forma paralela: surgieron en la década de 1980, se incubaron discretamente en la década de 1990 y florecieron en la década de los 2000, coincidiendo con los documentos de la Agenda 21 de la Cumbre de la Tierra de Río de Janeiro (1992). Ambos conceptos también formarán parte de las distintas reformas de la Política Agrícola Común (PAC) y más aún con la última reforma (2013) y la introducción de figuras profesionales como el "agricultor activo" o la introducción del pago verde o greening como complemento a las actividades tradicionales y en beneficio de iniciativas para conservar y poner en valor paisajes rurales de clara connotación cultural, patrimonial e identitaria (Silva, 2009; Costanza et al., 2017).

El regadío mantiene fuertes vínculos con ambos conceptos -servicios ecosistémicos y multifuncionalidad (Baskaran et al., 2013; Bommarco et al., 2013; Burton y Schwarz, 2013). El regadío y su gestión se viene debatiendo en base a la preocupación por el impacto social, económico y medioambiental que genera su desarrollo el nivel de participación de los regantes en la toma de decisiones (gobernanza) o la competencia existente entre la actividad agraria y otros usos del suelo y demandas del agua (Rutz et al., 2013; Prager, 2015). En este último caso, el agua es concebido como un recurso biofísico y social que se gestiona como un ciclo hidrosocial (Boelens et al., 2016; Ricart et al., 2018). Con ello se da un mayor protagonismo a las cuestiones políticas, socioeconómicas y administrativas del nexo regadío-sociedad y cobra interés la participación de los usuarios finales en los procesos de toma de decisiones (Caniglia et al., 2016). Un interés afín a los postulados de la gobernanza adaptativa y la ecología política como enfoques capaces de comprender las estructuras de poder y la complejidad que supone gestionar recursos naturales entre distintos intereses o demandas de uso (Karali et al., 2014; Loft et al., 2015). En este sentido, para la ecología política, la gestión del recurso agua no es una cuestión técnica y objetiva puesto que incluye su naturaleza social, involucrando valores, comportamientos y organizaciones político-económicas (Aubriot et al., 2018). 
La hipótesis de partida del presente artículo es la consideración de los parques naturales agrarios como una herramienta de gestión y gobernanza óptima en beneficio de la multifuncionalidad rural, especialmente en contextos donde conviven diversidad de intereses y valores sobre el trinomio regadío-medio ambiente-sociedad. Con ello, este artículo tiene por objetivo 1) identificar las cuestiones que generan mayor preocupación y debate entre los principales actores del sistema El Hondo-Los Carrizales y 2) analizar el nivel de gobernanza en la toma de decisiones que emana de su gestión. Ambos aspectos interaccionan en un caso de estudio concreto, el Parque Natural Agrario Los Carrizales y el corredor agroecológico que conforma con el Parque Natural El Hondo (Elche, Alicante).

\section{GeSTIÓN, GOBERNANZA Y ECOLOGÍA POLÍTICA: SUMANDO ACTORES PARA ASUMIR NUEVOS RETOS}

El recurso agua es diferente de otros elementos naturales, ya que no solo se utiliza para numerosos fines económicos y técnicos, sino que también tiene dimensiones culturales, sociales y simbólicas -dimensiones que se intensifican en ámbitos que sufren escasez hídrica y/o situaciones de sequía (Boelens, 2014). Ello repercute en la forma de asumir y superar problemáticas o retos futuros, de manera que las propuestas para resolver estos retos no dependan solamente de la disponibilidad o escasez de agua (Perramond, 2018), sino también de aspectos como el modelo de gestión del agua (Franzen et al., 2015); la competencia entre usos y la capacidad institucional para establecer acuerdos y convenios (Forouzani et al., 2013); las condiciones sociopolíticas y los requisitos ambientales prevalecientes que afectan los procesos y prácticas de planificación, desarrollo y gestión (Page y Bellotti, 2015); la adecuación e implementación de los marcos legales y regulatorios en materia de agua (Yao et al., 2017); las concesiones de agua y los costes asociados (Lika et al., 2017); la capacidad de inversión en infraestructuras (Levidow et al., 2014); las condiciones climáticas, sociales y ambientales de la matriz rural (Jafary y Bradley., 2018); la innovación tecnológica (Chartzoulakis y Bertaki, 2015); las actitudes y percepciones de los usuarios ante el consumo de agua (Marcantonio et al., 2018); o el nivel de gobernanza y transparencia en la toma de decisiones (Gupta et al., 2013).

La gestión de los recursos hídricos es una tarea especialmente desafiante en la que se requiere gestionar las complejidades en paralelo al factor social y cultural del agua (Lang et al., 2012). Si bien durante el siglo XX, el paradigma hidráulico justificó la intervención pública en la gestión de los recursos hídricos en base al interés nacional (Mollinga, 2008), las crisis ecológicas que le prosiguieron (López-Gunn, 2009), así como la exacerbación de conflictos sociales a escala regional y local (Smidt et al., 2016), generaron un cambio de enfoque que a las puertas del siglo XXI, que se materializó en la apuesta por criterios basados en la sostenibilidad y la participación de la sociedad en todo aquello que afecta a los bienes 
comunes y, entre ellos, el agua. A resultas de ello, mientras el siglo XX fue la era de la gestión administrativa y los paradigmas hidráulicos basados en el incremento de la oferta de agua, el siglo XXI simboliza la apuesta por la gestión de la demanda, la participación social y la consagración de la ecología política (Jacobs y Buijs, 2011; del Moral, 2009). En este sentido, enfoques como la gestión integrada de los recursos hídricos y la gobernanza de los bienes comunes complementan los postulados que abogan por incorporar a la gestión tradicional del agua la gama de sistemas políticos, sociales, económicos y administrativos que desarrollan y gestionan esos recursos hídricos como respuesta a la consecución de los retos de la sociedad (Munaretto et al., 2014). Sin embargo, cabe diferenciar entre gestión y gobernanza, puesto que, si bien la gestión se centra en el desarrollo de actividades específicas para alcanzar unos objetivos concretos, ya sea desde la autogestión o las alianzas público-privadas (Wehn et al., 2015; Anderson et al., 2016), la gobernanza incluye los procesos de toma de decisiones, así como la definición de objetivos entre una variedad de intereses. Al involucrar a las partes interesadas, la gobernanza participativa facilita la articulación de prioridades, permite ejercer derechos legales, satisfacer sus necesidades y mediar en las diferencias entre usuarios (Sinclair et al., 2013; Varis et al., 2014; Gutzler et al., 2015).

El cambio de paradigma hídrico (de la gestión a la gobernanza) es uno de los avances más notables en la ciencia social contemporánea (Howlett et al., 2009). Ello ha supuesto evolucionar desde los postulados jerárquicos (top-down) hacia la colaboración en red (bottom-up), capaz de integrar la toma de decisiones y reducir la distancia entre los intereses públicos (servicios ecosistémicos, multifuncionalidad) y los beneficios privados (productividad del regadío). Sin embargo, la gestión y la gobernanza no son conceptos mutuamente excluyentes (Lubell y Edelenbos, 2013; Moore, 2013). La gestión implica incorporar actitudes originarias de la gobernanza, como son afrontar la incertidumbre, aceptar la negociación, impulsar la deliberación o aumentar la sensibilidad hacia los sistemas socio-ecológicos (Hileman et al., 2016). Según Armitage et al. (2012), el reconocimiento de las similitudes y diferencias entre la gestión y la gobernanza del regadío es crucial dada la naturaleza compleja, no lineal y de escala que emana de los desafíos socio-ecológicos que conviven en la era del cambio ambiental global. Existen varias definiciones de gobernanza, pero todas se relacionan con la gama de actores y estructuras movilizados en la formulación e implementación de políticas del agua (Schulz et al., 2017). Según la OCDE (2015), la efectividad, la eficiencia y la confianza/compromiso son los tres principios clave que reconoce la gobernanza del agua. El primero se relaciona con la contribución de la gobernanza para definir objetivos y metas sobre políticas de agua sostenibles en todos los niveles de gobierno y gestión. El segundo se centra en la contribución de la gobernanza a la maximización de los beneficios que genera la gestión sostenible del agua y el bienestar social al menor coste posible. Por último, el tercero se refiere a la contribución de la gobernanza para generar confianza pública y garantizar la inclusión de las partes interesadas a través de la legitimidad democrática y la equidad social. 
De hecho, los acuerdos de gobernanza a menudo se juzgan en función de su capacidad para superar tensiones o conflictos entre las partes interesadas y una vez identificadas distintas relaciones de poder (Araral y Wang, 2013; Akhmouch y Clavreul, 2016).

Un enfoque propuesto para superar estas tensiones es la gestión participativa del regadío, en la que se combina la máxima eficiencia en la asignación y distribución del agua con la participación de los actores en todas las fases de su gestión, como son la planificación, la operación, el mantenimiento, el seguimiento y la evaluación de los procesos (Das et al., 2015). Este paso desde un enfoque top-down y tecnocrático a un enfoque bottom-up y sociocrático se fundamenta en una mayor concienciación acerca de la complejidad de las problemáticas actuales en torno al recurso agua y la apuesta por soluciones integradas y planificadas con y para los usuarios finales (Ricart y Clarimont, 2016). De hecho, las cuestiones sobre quién forma parte de la toma de decisiones y en qué medida ejerce cierto control o influencia sobre el conjunto de intereses ocupan el centro del debate sobre la legitimidad institucional de la gestión del agua en general y de los sistemas de regadío en particular (Obermeister, 2017).

Tabla 1. Listado y tipología de actores entrevistados en el sistema El Hondo-Los Carrizales

\begin{tabular}{|c|c|}
\hline $\begin{array}{l}\text { Administración } \\
\text { - } \quad \text { Parque Natural El Hondo } \\
\text { - } \quad \text { Confederación Hidrográfica del Segura }\end{array}$ & $\begin{array}{l}\text { Intereses agrícolas } \\
\text { - } \quad \text { Comunidad General de Regantes Riegos de } \\
\text { Levante Margen Izquierda } \\
\text { - } \quad \text { Parque Natural Agrario Carrizales/Comunidad de } \\
\quad \text { Regantes de Carrizales } \\
\text { - } \quad \text { ASAJA (Elche) } \\
\text { - } \quad \text { La Unió de Llauradors i Ramaders (Alacant) }\end{array}$ \\
\hline $\begin{array}{l}\text { Intereses ambientales } \\
\text { - } \quad \text { Agrupación Rural Sur de Alicante } \\
\text { - } \quad \text { SEO-BirdLife (Alicante) } \\
\text { - } \quad \text { Asociación de Naturalistas del Sureste } \\
\text { - } \quad \text { Amigos de los Humedales del Sur de Alicante } \\
\text { - } \quad \text { Observatorio del Vinalopó }\end{array}$ & $\begin{array}{l}\text { Intereses sociales/académicos } \\
\text { _ } \quad \text { Associació pel Desenvolupament Rural del Camp } \\
\text { d'Elx } \\
\text { - } \quad \text { Asociación para el Desarrollo Sostenible de los } \\
\quad \text { Espacios Naturales Protegidos } \\
\text { - } \quad \text { Foro Pro Agua } \\
\text { - } \quad \text { Ajuntament d'Elx } \\
\text { - } \quad \text { Ajuntament de Crevillent }\end{array}$ \\
\hline
\end{tabular}

\section{Metodología y datos}

El análisis del caso de estudio ha requerido el uso de técnicas de naturaleza cualitativa tales como entrevistas semi-estructuradas y cuestionarios (Caniato et al., 2014; Montgomery et al., 2016). Ambas herramientas se han aplicado a los representantes de la administración y los intereses agrícolas, ambientales y sociales/académicos que conviven en el sistema El 
Hondo-Los Carrizales. Para la realización de las entrevistas se partió de una lista inicial de actores en base a la información disponible en los planes de gestión agrícola de la región. Así mismo y tal y como plantean Kharel et al. (2018) en su artículo, se confirmó la lista inicial por parte de expertos en la materia, informantes locales e investigadores afines a la temática de estudio. Se identificaron un total de 16 actores colectivos (Tabla 1). Todos ellos fueron entrevistados por el primer autor entre los meses de abril y septiembre de 2017. Cada entrevista tuvo lugar en la sede de la institución/organismo o en un espacio público, con el máximo responsable o el técnico experto en la materia y con una duración de entre 45 y 120 minutos. Todas las entrevistas fueron audio grabadas. El guion de la entrevista se estructuró en tres bloques: 1) Características, valores y funciones asociadas al Parque Natural El Hondo, 2) Gestión del parque natural e interacción con el Parque Natural Agrario Los Carrizales y 3) Problemáticas y retos que comporta la gestión del ámbito. La entrevista se complementó con la realización de un cuestionario sobre el nivel de gobernanza existente en la toma de decisiones y las relaciones de poder identificados en el corredor agroecológico El Hondo-Los Carrizales. El cuestionario consta de tres bloques y 14 preguntas (ver Apéndice): a) nivel de representación en el modelo de gestión del parque y factores explicativos, b) valoración del rol del resto de actores y c) predisposición para establecer alianzas y capacidad para alcanzar acuerdos. Algunas de las preguntas con opciones de respuesta bidireccional han requerido la utilización de una escala psicométrica tipo Likert (Kampen, 2019).

\section{El Parque Natural Agrario los Carrizales Y EL CORREDOR AGROECOLÓGICO CON El HoNdo}

\section{Localización y origen}

El Parque Natural Agrario Los Carrizales ocupa una extensión de 13 km² y se sitúa en el área que ocupaba el antiguo Golfo de Elche (Abellán, 2019). Este parque agrario y natural se localiza en el área periurbana de la ciudad de Elche y está ubicado hidrológicamente en la Vega Baja del Segura -aunque en término ilicitano-, entre el Parque Natural El Hondo y El Parque Natural de las Salinas de Elche-Santa Pola (Ruiz, 2014), para constituir el nexo físico de unión y verdadero corredor ecológico entre ambos humedales (Guilló y Montaner, 2014). (Figura 1). La connotación agraria de este ámbito tiene su origen histórico en los procesos de desecación y bonificación iniciados con la Real Cédula de 1748 concedida por el rey Fernando VI al duque de Arcos (Canales, 2004). 


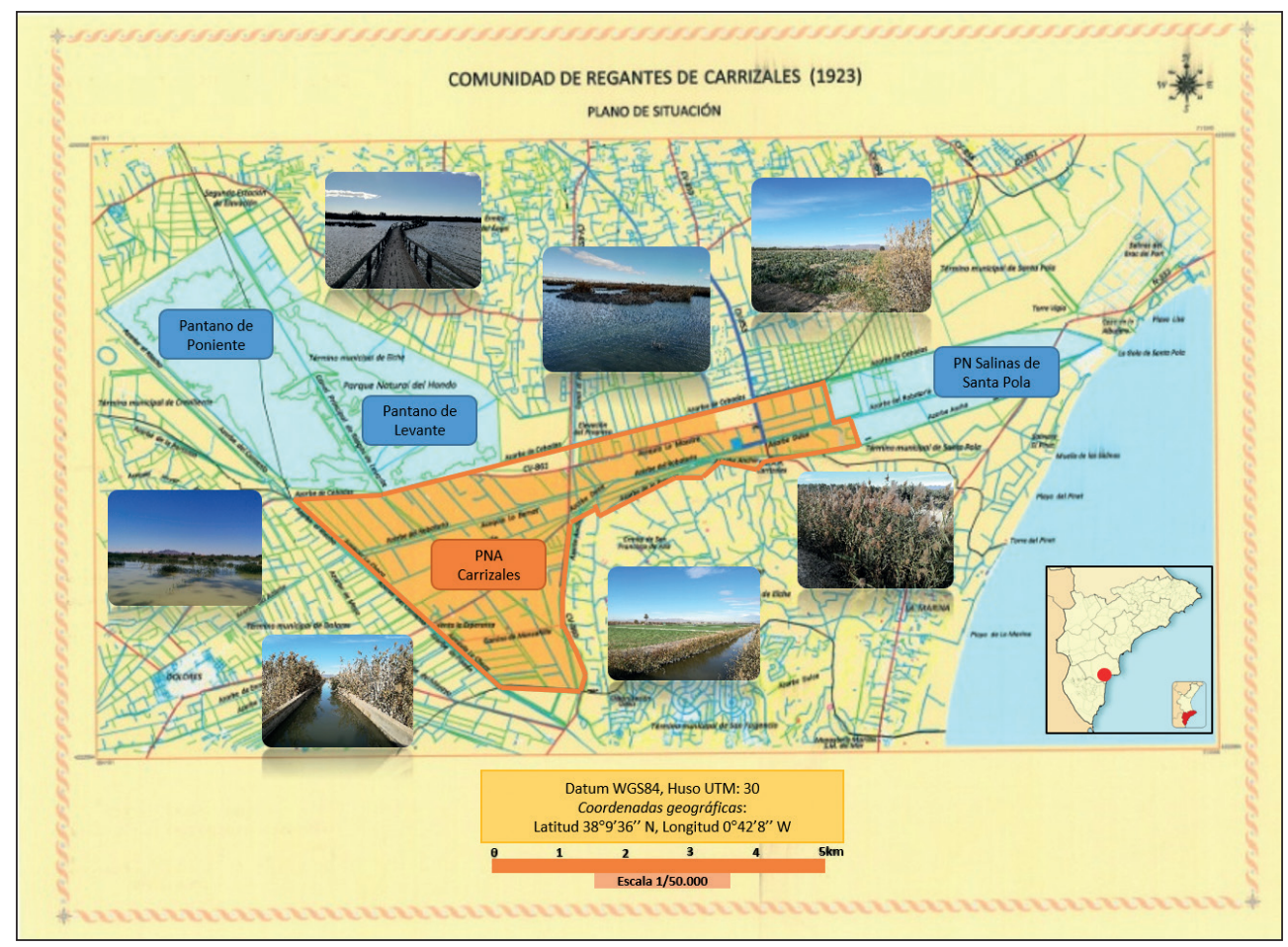

Figura 1. Situación y ámbito colindante del Parque Natural Agrario Los Carrizales Fuente: Comunidad de Regantes de Carrizales. Adaptado de Segrelles (2013).

\section{Historia y objetivos}

Este parque es una iniciativa surgida a finales del año 2008 a petición de la Comunidad de Regantes de Carrizales, entidad de derecho público sin ánimo de lucro adscrita a la Confederación Hidrográfica del Segura y que cuenta con cerca de 400 agricultores. La propuesta se conceptualizó en base a la experiencia del Parque Agrario del Baix Llobregat (Barcelona), creado en 1998 con el fin de gestionar de forma integrada los usos productivos agrarios y las funciones ambientales del Delta del río Llobregat -este espacio de 3.300 ha cuenta con humedales que ejercen una función clave en la ruta migratoria euroafricana de las aves acuáticas-, mientras que su plasmación y desarrollo se benefició del proyecto WADI, desarrollado entre 2006 y 2008 dentro del sexto programa marco europeo con el objetivo de identificar los valores y potencialidades del sistema de El Hondo y su área de influencia y promover, con ello, escenarios de futuro sostenibles basados en la gestión integrada de los aspectos ecológicos, culturales y socioeconómicos que determinan el funcionalismo del ámbito (Cantarino, 2010). Así mismo, el proyecto afianzó el entendimiento entre regantes, organizaciones am- 
bientales y administración en beneficio de la gestión conjunta del espacio y la convivencia entre la protección de los valores naturales, culturales y paisajísticos, la actividad rural y el uso social del territorio. Se impulsa así la viabilidad del territorio como zona económicamente funcional y ecológicamente protegida (Figura 2).

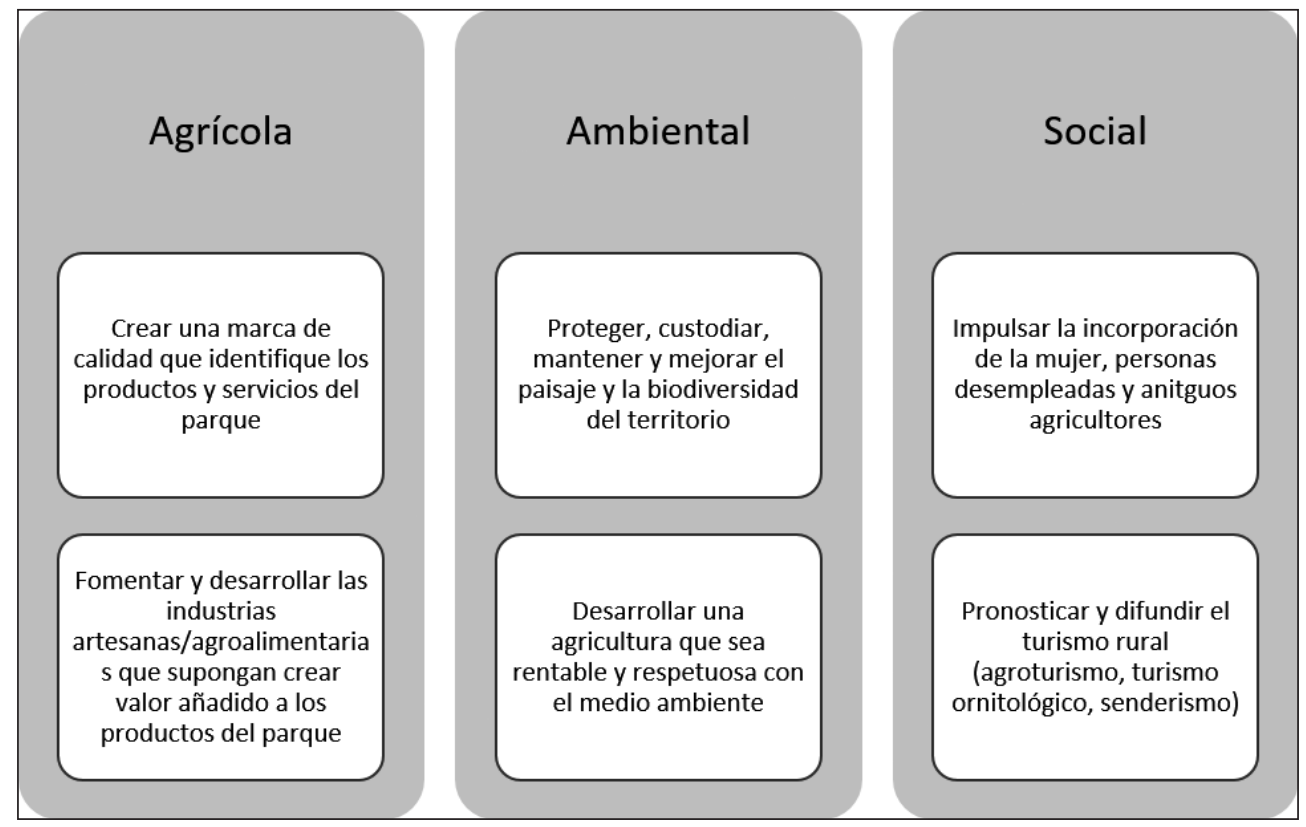

Figura 2. Principales objetivos del parque natural agrario.

Fuente: Página web de la Comunidad de Regantes de Carrizales.

\section{Gestión}

Mientras la Generalitat Valenciana, a través de la figura del Parque Natural El Hondo (1988), es el órgano gestor de la conservación del humedal del Hondo (De la Vega et al., 2010), la Comunidad General de Regantes Riegos de Levante Margen Izquierda es el propietario de la principal masa de agua del Parque Natural el Hondo y la Comunidad de Regantes de Carrizales gestiona el ámbito colindante donde se ubica el Parque Natural Agrario Los Carrizales. La misión de la Comunidad de Regantes de Carrizales se centra en la distribución, tutela y reutilización de las aguas concedidas con fines agrícolas y ambientales, así como en el mantenimiento de sus infraestructuras, ya sean embalses reguladores de riego o acequias y azarbes de distribución de aguas sobrantes, algunas de las cuales son compartidas con la Comunidad General de Regantes Riegos de Levante Margen Izquierda (Gil Olcina, 2016). El modelo de gestión integrada del ámbito ha sido reconocido con el Premio Custodia del 
Territorio 2016, otorgado a la Asociación de Naturalistas del Sureste (ANSE) y Amigos de los Humedales del Sureste de Alicante (AHSA) y a la Comunidad de Regantes de Carrizales en la III Edición de los Premios Biodiversidad y Sociedad que concede la Generalitat Valenciana por su trabajo en la conservación de la biodiversidad y el mantenimiento y fomento de las actividades agrícolas en el humedal de Los Carrizales y el entorno del Parque Natural del Hondo mediante acuerdos de custodia.

\section{Infraestructuras y sistema de riego}

La mayor parte de la superficie agraria de Los Carrizales es de regadío, siendo el riego superficial por inundación el sistema dominante. La misión secular de la comunidad de regantes se ha centrado en el mantenimiento y conservación óptima de sus infraestructuras, formadas fundamentalmente por una red hídrica de más de 200 kilómetros en la que destacan los azarbes, acueductos y canales al aire libre, sin cementar ni entubar, al contrario de lo que sucede en otros lugares próximos (Segrelles, 2013). El actual sistema de riego del parque forma parte de la red de irrigación de la huerta del Bajo Segura y Bajo Vinalopó que, debido a las condiciones de alta aridez que condicionan el SE ibérico, se ha ido construyendo históricamente sobre el principio básico de la máxima reutilización del agua (Martínez-Pérez et al., 2009). Así mismo, y dadas las condiciones de relieve y subsuelo de la vega del Segura, el sistema necesita de dos sistemas de canalizaciones paralelos: uno de irrigación (aguas vivas) y otro de drenaje (aguas muertas) (Trapote et al., 2015). Este último asume la función de desembocadura del tramo final del río Vinalopó puesto que este carece de un cauce propio para su salida autónoma al mar y aprovecha el azarbe de Cebadillas. El parque natural agrario, ubicado dentro del "Sistema Vinalopó-Alacantî" adscrito a la Confederación Hidrográfica del Júcar, recibe aguas de dos orígenes, por una parte, de la cuenca hidrográfica del Segura -las que le corresponde por vía concesional-, y por otra parte, de la demarcación del Júcar -las que por vía de hecho son vertidas en sus canales y azarbes fruto del perímetro que comparte con los dos parques naturales (El Hondo y Salinas de Elche-Santa Pola).

\section{Principales cultivos, ganadería y marca de calidad}

En las cerca de 1.300 ha que conforman el parque natural agrario se distinguen diversos aprovechamientos, desde hortalizas, cereales y frutas hasta cultivos arbóreos. Cabe decir que cerca del 40\% de la extensión total del área del parque no está cultivada (Segrelles, 2013). Los cultivos emblemáticos del parque son el Melón del Carrizal, que ocupa una superficie aproximada de 13 ha rotadas anualmente -por lo que el cultivo del melón ocupa de manera efectiva el doble o triple de superficie- y la granada Mollar de Elche -con cerca de una tercera parte de la superficie destinada al melón-, ambos valorados en los mercados de proximidad e internacionales. De hecho, este último cuenta con el sello Denominación de Origen Protegida (DOP). También las parcelas se rotan con cultivos forrajeros, entre los que destaca la 
alfalfa, que aportan alimento a las más de cinco mil cabezas de ganado caprino y ovino que pastorean por el parque.

\section{Medio ambiente y multifuncionalidad}

Tras su inclusión en la lista de espacios naturales protegidos por virtud de la Ley 11/1994, de 27 de diciembre de Espacios Naturales Protegidos de la Generalitat Valenciana, este ámbito de transición se considera un ámbito de interés por su función conectora y de especial protección agrícola al conectar y vertebrar ambos parques naturales (El Hondo y Las Salinas de Elche-Santa Pola) mediante la malla de azarbes y canales de Los Carrizales (Niño et al., 2015). Así se refleja en la planificación urbanística municipal al señalar la delimitación del suelo no urbanizable protegido de zonas húmedas, cauces y barrancos (Artículo 7.7) del Plan General de Crevillent de 2011) o la servidumbre de protección de ambos parques naturales (Artículo 183 apartado D) y el régimen específico de ambos espacios al ser considerados como parajes naturales (Artículo 223) del Plan General de Elche de 1998 (texto refundido de 2014). El ámbito también está catalogado como Zona Húmeda de Especial Interés al ser incluida en el Catálogo de Zonas Húmedas de la Comunitat Valenciana como zona n ${ }^{\circ} 13$ (2002). Además, parte del parque natural agrario se incluye en el perímetro del Parque $\mathrm{Na}$ tural de Las Salinas de Elche-Santa Pola $\left(26 \mathrm{~km}^{2}\right)$ y de la zona perimetral de protección del Parque Natural El Hondo $\left(24 \mathrm{~km}^{2}\right)$, ambos reconocidos como Humedales de Importancia Internacional (Zonas Ramsar) y en virtud de la Ley 42/2007, de 13 de diciembre, del patrimonio natural y de la biodiversidad por la que se establecen los espacios protegidos de la Red Natura 2000, también cuentan con la designación como Zonas de Especial Protección para las Aves (ZEPA) (código ES0000058) conforme a la Directiva Europea 79/409/CEE relativa a la Conservación de las aves silvestres, y Lugar de Importancia Comunitario (LIC) (código ES0000058) en aplicación de la Directiva Hábitat 92/43/CEE. En 2016, se incluyeron las zonas húmedas catalogadas de Los Carrizales en la ZEPA de El Hondo, medida demandada años atrás tanto por los grupos ecologistas AHSA y ANSE como por parte de la Comunidad de Regantes de Carrizales. Por su riqueza ornitológica e indudable valor ambiental y paisajístico, este singular espacio natural recibió un especial tratamiento legal y protección a nivel regional tras su inclusión en el Catálogo Valenciano de Zonas Húmedas (2010) dentro del grupo "Albuferas y marjales litorales". Asimismo, en el Plan de Ordenación de Recursos Naturales (PORN) del Sistema de Zonas húmedas del Sur de Alicante del mismo año -considerado como Proyecto Medioambiental Estratégico conforme al Decreto 31/2010, del Consell-, se define a Los Carrizales como "Área de Interés Especial Natural en la Zona Periférica de Protección de los Parques Naturales de El Hondo y de las Salinas de Santa Pola" (Abellán, 2019). Con el fin de recrear el ecosistema natural del ámbito en 2005 la organización ecologista AHSA adquirió una parcela de $15.000 \mathrm{~m}^{2}$ en la zona de Manzanilla que supuso la creación de isletas para la nidificación de las aves acuáticas. En 2009 y en pa- 
ralelo a la gestación e impulso del proyecto de parque natural agrario, se inicia el proyecto de custodia del territorio "Humedal de los Carrizales" de la mano de la comunidad de regantes y las organizaciones ecologistas ANSE y AHSA para recrear el ecosistema original del ámbito mediante la creación de varias lagunas de aguas permanentes para favorecer la presencia de aves endógenas como la malvasía cabeciblanca (Oxiura leucocephala) y la cerceta pardilla (Marmaronetta angustrirostris) ambos en peligro de extinción, y de peces como el mújol (Mugil cephalus) y el fartet (Aphanius iberus).

Más recientemente y en el marco de los acuerdos de custodia del territorio ya vigentes, se ha planteado la posibilidad de promover diversos corredores verdes con fines lúdico-educativos que permitan conectar los dos parques naturales con el parque natural agrario.

\section{Resultados}

\section{Principales preocupaciones}

Las preocupaciones y los retos que debe afrontar tanto la gestión del Parque Natural Agrario Los Carrizales como del corredor agroecológico que conforma con el Parque Natural El Hondo responden a una gran diversidad y complejidad temática, tanto a nivel técnico (salinidad, calidad del agua regenerada, provisión de servicios ecosistémicos) como funcional (competencia entre usos de agua, relevo generacional, participación en la toma de decisiones). Dos ideas clave han sido reconocidas por parte de los 16 entrevistados: 1) la dificultad que supone otorgar un valor simbólico al mantenimiento del paisaje y la generación de servicios ecosistémicos como factores de desarrollo local intrínsecos a la práctica agrícola, reconocidos socialmente y compensados económicamente y 2) la falta de apoyo administrativo y técnico para que el corredor El Hondo-Los Carrizales sea contemplado como un espacio de aprendizaje continuo y un laboratorio de ensayo de los retos que debe afrontar el binomio regadío-humedal. Ambas constataciones ponen de manifiesto la necesidad de profundizar en el proceso de toma de decisiones y el nivel de gobernanza existente en el modelo de gestión del ámbito.

\section{Nivel de gobernanza}

Del análisis de las respuestas aportadas mediante el cuestionario realizado a los diferentes actores cabe destacar la interrelación entre tres aspectos. En primer término, si bien el Parque Natural Agrario Los Carrizales no forma parte ni está representado en la Junta Rectora del Parque Natural El Hondo, la Comunidad de Regantes de Carrizales reconoce su interés por integrarse en los procesos de toma de decisiones en todo lo que confiere el corredor agroecológico, puesto que ambos parques comparten intereses (agrícolas y ambientales) y retos (salinidad, disponibilidad de agua, cambio climático) a corto y medio plazo. Se evidencia, además, la necesidad de establecer mecanismos de gestión específicos que aseguren la repre- 
sentación y consideración del parque natural agrario en aquellas decisiones que pueden afectar directa o indirectamente el desarrollo de Los Carrizales. Sirvan de ejemplo las cuestiones relacionadas con las infraestructuras compartidas con el Parque Natural El Hondo (zonas perimetrales, disponibilidad y calidad del agua, estado de los azarbes) o las estrategias para marcar tendencia o ser referente en materia de convivencia entre productividad agrícola y protección ambiental (nuevas lagunas, acuerdos bilaterales con organizaciones ecologistas). Sin embargo, la Comunidad de Regantes de Carrizales remarca la falta de reconocimiento administrativo y técnico que le otorgan el resto de actores del ámbito, hecho que supone una limitación evidente a la hora de gestionar ambos aspectos.

Segundo, la Comunidad de Regantes de Carrizales se considera indispensable en el modelo de gestión del corredor agroecológico El Hondo-Los Carrizales al igual que reconoce esa consideración al ente gestor del Parque Natural El Hondo, la Confederación Hidrográfica del Segura y la Comunidad General de Regantes Riegos de Levante Margen Izquierda (que reúne una superficie cercana a las 20.000 ha y 8 entidades de base). Así mismo, la Comunidad de Regantes de Carrizales también reconoce la necesidad de contar con la implicación de los ayuntamientos de Elche y Crevillent así como de las organizaciones ecologistas SEO/ BirdLife y ANSE en todo aquello relacionado con la multifuncionalidad del regadío y la convivencia entre agricultura y naturaleza. Por el contrario, justifica el rol complementario del resto de actores (sindicatos agrícolas, organizaciones ecologistas, Observatorio del Vinalopó, agrupación rural) así como el carácter prescindible del Foro Pro Agua por tratarse de actores o bien no directamente afectados por las decisiones sobre la gestión directa del corredor agroecológico o bien por tratarse de actores sin ninguna responsabilidad a la hora de establecer convenios de colaboración entre intereses dispares. Desde el punto de vista inverso, a saber, qué consideraciones recibe la Comunidad de Regantes de Carrizales por parte del resto de actores, cabe destacar como 8 de los 15 encuestados reconocen su rol indispensable en la promoción de una gestión holística del paraje (entre ellos, el ente gestor del Parque Natural El Hondo, la Comunidad General de Regantes Riegos de Levante Margen Izquierda, las organizaciones ecologistas y de desarrollo rural o el Ayuntamiento de Elche). Esta valoración se complementa con la valoración del rol que ejerce cada actor en la toma de decisiones que afectan o pueden afectar al corredor agroecológico y que muestra como la Comunidad de Regantes Los Carrizales se auto-valora con una puntuación media ( 3 en una escala de 5 puntos Likert) mientras que valora de forma muy positiva el rol de ANSE en base a sus acuerdos de custodia del territorio o hace patente la valoración claramente negativa de la Confederación Hidrográfica del Segura debido a su falta de implicación en las cuestiones hidrológicas e hidráulicas que afectan al Parque Natural Agrario Los Carrizales. También valora negativamente la actitud de los sindicatos agrícolas, el Foro Pro Agua y al Observatorio del Vinalopó al considerar que no ofrecen un discurso claro en cuanto al futuro del ámbito se refiere. 
Tercero, la totalidad de los actores priorizan la definición de acuerdos con el resto si bien difieren en justificar los aspectos que motivan ese acuerdo. Con ello, si bien el conjunto de los actores -a excepción de la Comunidad General de Regantes Riegos de Levante Margen Izquierda y el ente gestor del Parque Natural El Hondo- coinciden en destacar la predisposición de los interesados como el factor clave en la consecución de acuerdos, otros actores como la Confederación Hidrográfica del Segura, las organizaciones ecologistas o las asociaciones para el desarrollo rural coinciden en valorar positivamente la compatibilidad entre discursos o la afinidad entre retos a afrontar, mientras los sindicatos agrícolas o los ayuntamientos creen necesario establecer una estrategia común para poder identificar acuerdos potenciales. En cuanto al marco temporal y la forma que toman dichos acuerdos, dominan los convenios de carácter puntual (sobre todo entre comunidades de regantes, sindicatos agrícolas o ayuntamientos), si bien se tiende a combinarlos con acciones que requieren un marco de acción permanente, por ejemplo, en casos en los que se requiera establecer diagnósticos, proyectos o actuaciones de relevancia (mantenimiento de infraestructuras, calidad del agua o recuperación de hábitos faunísticos). También se utilizan notas o comunicados de prensa para publicitar las actividades desarrolladas o los acuerdos previstos. Por último y en relación a los factores que impiden la promoción de acuerdos entre intereses dispares, la mayor parte de los actores (11 de los 16, el 69\%, entre ellos los integrantes de la Administración, los sindicatos agrícolas, la Comunidad de Regantes de Carrizales, parte de los intereses ambientales -AHSA, ANSE y Observatorio del Vinalopó- parte de los intereses sociales -ayuntamientos y Foro Pro Agua-) coinciden en el diagnóstico: tanto la incompatibilidad de discursos como la poca predisposición de las partes para argumentar sus posturas impiden acuerdos fructíferos. Otros aspectos como la falta de una estrategia común o el trato de favor -entendido como una forma de favorecer, beneficiar o privilegiar los intereses de alguno de los actores en detrimento del resto- también dificultan la negociación entre iguales, tal y como apuntan ambas comunidades de regantes, SEO/Birdlife o la Associació pel Desenvolupament Rural del Camp d'Elx. Otros aspectos como la defensa de intereses partidistas o la falta de implicación política son aspectos que identifica el Foro Pro Agua como limitaciones en la garantía de éxito del proceso de toma de decisiones.

\section{Discusión y Conclusiones}

Los conflictos socio-ambientales están aumentando a nivel mundial (Couix y Gonzalo-Turpin, 2015). La literatura sobre la conservación de los recursos naturales y la gestión ambiental de espacios antrópicos destaca dos factores importantes que condicionan el éxito con el que se pueden abordar estos conflictos. Primero, la correlación entre las percepciones de los directamente afectados y los indirectamente interesados influye en la gestión directa de los sistemas y la búsqueda de soluciones compartidas (Booth y Halseth, 2011). Segundo, es esencial que los procesos participativos aborden las consecuencias ecológicas, económicas 
y sociales de diferentes alternativas de gestión de los sistemas hidrosociales de forma integrada, porque a menudo las relaciones de poder y los intereses de los actores responden a objetivos de gestión dispares y/o confrontados (Castro et al., 2016). Tanto el Parque Natural El Hondo como el Parque Natural Agrario Los Carrizales responden a la histórica interacción entre ecosistema y la acción transformadora del hombre con fines orientados hacia una mayor salubridad y eficacia agrícola (Canales et al., 2012). Dicha interacción ha facilitado el desarrollo de un ámbito multifuncional que requiere de la aplicación de los postulados de la gobernanza y la ecología política para promover o afianzar acuerdos entre intereses dispares. Sin embargo, el fomento de la participación no es, per se, garantía de éxito (Hoolohan et al., 2018). El análisis del comportamiento y la percepción de los discursos que confluyen en todo proceso de gestión participativa es clave a la hora de, por ejemplo, abordar si la capacidad de gestionar la multifuncionalidad es individual o colectiva (Beltrán y Velázquez, 2017). En este sentido, la mayor parte de los encuestados - con la Comunidad de Regantes de Carrizales o las organizaciones ecologistas AHSA, ANSE y SEO/Birdlife a la cabeza- son partidarios de una gestión colectiva del corredor agroecológico para con ello aprovechar las sinergias creadas a nivel ambiental, socioeconómico, cultural y paisajístico. Para ello, abogan por superar las barreras administrativas y afianzar un modelo de gestión integrada y adaptativa basada en el aprendizaje a través de la práctica -tal y como han podido comprobar en los acuerdos de custodia del territorio. Es lo que se viene aplicando como "learning by doing" (Conallin et al., 2018). En respuesta a la hipótesis de partida formulada, a saber, que los parques naturales agrarios, como piezas clave de corredores agroecológicos, son una herramienta de gestión y gobernanza óptima que favorece la multifuncionalidad rural, el caso de estudio se debe gestionar como un sistema de aprendizaje que dé respuesta a la complejidad y la incertidumbre, así como afronte las necesidades particulares de los actores que conviven en el corredor agroecológico El Hondo-Los Carrizales. Sin embargo, ello requiere de la integración de todas las partes interesadas en la gestión de las problemáticas (salinidad, calidad del agua regenerada, gestión de los azarbes, perímetro y figuras de protección ambiental) y retos comunes (provisión de servicios ecosistémicos, competencia entre usos de agua, preservación de la actividad agrícola y apoyo generacional, promover el reconocimiento social del ámbito), así como la evaluación de los hitos conseguidos hasta el momento, como los acuerdos de custodia del territorio, si bien se trata exclusivamente de acuerdos entre partes y no así de acuerdos colectivos que agrupen a la totalidad de los actores del ámbito, como sería en el caso de promover un mecanismo oficial de gestión integrada del corredor agroecológico El Hondo-Carrizales. Con la finalidad de promover la gestión integrada del corredor agroecológico, se plantean algunas actuaciones en pro de mejorar la gobernanza en la toma de decisiones y en beneficio del carácter multifuncional del corredor agroecológico: 1) definir un plan de gestión específico para el corredor agroecológico que incluya al conjunto de actores aquí descritos -definiendo la responsabilidad de cada actor y asegurando su interrelación- y que afiance el rol de éste ámbito multifuncional -con especial hincapié a su función ambiental como barrera 
para la intrusión marina o reduciendo la vulnerabilidad frente episodios de sequía percibidos como recurrentes fruto de los impactos del cambio climático-; 2) afianzar el reconocimiento de los productos emblemáticos del Parque Natural Agrario Los Carrizales bajo una "marca territorio" (Hernández, 2008) y promover el sello Indicación Geográfica Protegida (IGP) del Melón del Carrizal como motor de desarrollo rural y venta de proximidad; y 3) definir una estrategia de visualización recreativa y turística del corredor agroecológico como herramienta de socialización de ambos parques mediante el diseño de rutas paisajísticas o jornadas de divulgación.

\section{ReFERENCIAS}

Akhmouch, A. y Clavreul, D. (2016): Stakeholder engagement for inclusive water governance: "Practicing what we preach" with the OECD Water Governance Initiative. Water 8, 204-220.

Anderson, M.B., Hall, D.M., McEvoy, J., Gilbertz, S.J., Ward, L. y Rode, A. (2016): Defending dissensus: Participatory governance and the politics of water measurement in Montana's Yellowstone River Basin. Environmental Politics 25(6), 991-1012.

Araral, Y. y Wang, Y. (2013): Water Governance 2.0: A review and second generation research agenda. Water Resources Management 27, 3945-3957.

Armitage, D., DE Loë, R. y Plummer, R. (2012): Environmental governance and its implications for conservation practice. Conservation Letters 5, 245-255.

Aubriot, O., Fernández, S., Trottier, J. y Fustec, K. (2018): Water technology, knowledge and power. Addressing them simultaneously. WIREs Water 5, e1261.

Baskaran, R., Colombo, S. y Cullen, R. (2013): Public preferences in irrigation and conservation development projects: Does simultaneous consideration of substitutes in choice sets matter? Land Use Policy 33, 214-226.

Beltrán, M.J. y Velázquez, E. (2017): The political ecology of water metabolism: The case of the Cobre las Cruces copper mine, southern Spain. Sustainability Science 12, 333-343.

Boelens, R. (2014): Cultural politics and the hydrosocial cycle: Water, power and identity in the Andean highlands. Geoforum 57, 234-247.

Boelens, R., Hoogesteger, J., Swyngedouw, E., Vos, J. y Wester, P. (2016): Hydrosocial

territories: A political ecology perspective. Water International 41(1), 1-14.

Bommarco, R., Kleijn, D. y Potts, S.G. (2013): Ecological intensification: harnessing ecosystem services for food security. Trends in Ecology Evolution 28, 230-238.

Booth, A. y Halsethh, G. (2011): Why the public thinks natural resources public participation processes fail: A case study of British Columbia communities. Land Use Policy 28, 898-906.

Burton, R.J.F. y Schwarz, G. (2013): Result-oriented agri-environmental schemes in Europe and their potential for promoting behavioural change. Land Use Policy 30, 628-641.

Canales, G. (2004): “Avenamiento y utilización de aguas muertas”, en Gil Olcina, A., La cultura del agua en la cuenca del Segura. Murcia, Fundación Cajamurcia, pp. 439-477. 
Canales, G., López, A. y Segrelles, A. (2012): Percepción de un Espacio Natural protegido en los municipios de su entorno: El caso de El Hondo de Elche. En Araque, E. et al. (ed.). Investigando en rural. Ulzama Ediciones, Pamplona, pp. 323-332.

Caniato, M., Vaccari, M., Visvanathan, C. y Zurbrügg, C. (2014): Using social network and stakeholder analysis to help evaluate infectious waste management: a step towards a holistic assessment. Waste Management 34, 938-951.

Caniglia, B., Frank, B., Kerner, B. y Mix, T.L. (2016). Water policy and governance networks: A pathway to enhance resilience toward climate change. Sociological Forum 31(S1), 828-845.

Castro, A.J., Vaughan, C.C., Julian, J.P. y García-Llorente, M. (2016): Social demand for ecosystem services and implications for watershed management. Journal of the American Water Resources Association 52(1), 209-221.

Charzoulakis, K. y Bertaki, M. (2015): Sustainable water management in agriculture under climate change. Agriculture and Agricultural Science Procedia 4, 88-98.

Conallin, J., Wilson, E., Campbell, J. (2018): Implementation of environmental flows for intermittent river systems: Adaptive management and stakeholder participation facilitate implementation. Environmental Management 61(3), 497-505.

Costanza, R., DE Groot, R., Braat, L., Kubiszewski, I., Fioramonti, L., Sutton, P., Farber, S. y Grasso, M. (2017). Twenty years of ecosystem services: How far have we come and how far do we still need to go? Ecosystem Services 28, 1-16.

Couix, N. y Gonzalo-Turpin, H. (2015): Towards a land management approach to ecological restoration to encourage stakeholder participation. Land Use Policy 46(1), 155-162.

Das, B., Singh, A., Panda, S.N y Yasuda, H. (2015): Optimal land and water resources allocation policies for sustainable irrigated agriculture. Land Use Policy 42, 527-537.

De la Vega, A., del Campo, F.J. y Agulló, A. (2010): Estrategia para la gestión ambiental en el Parque Natural de El Hondo (Alicante). Cuides: Cuaderno Interdisciplinar de desarrollo Sostenible 5, 79-94.

Del Moral, L. (2009): Nuevas tendencias en gestión del agua, ordenación del territorio e integración de políticas sectoriales. Scripta Nova. Revista electrónica de geografía y ciencias sociales 13, 281-309.

Duru, M., Therond, O., Martin, G., Martin-Clouaire, R., Magne, M.A., Justes, E., Journet, E.P., Aubertot, J-N., Savary, S., Bergez, J-E. y Sarthou, J.P. (2015): How to implement biodiversity based agriculture to enhance ecosystem services: a review. Agronomy for Sustainable Development 35, 1259-1281.

Forouzani, M., Karami, E. y Zamani, G.H. (2013): Agricultural water poverty: using Q-methodology to understand stakeholders' perceptions. Journal of Arid Environments 97, 190-204.

Franzen, F., Hammer, M. y Balfors, B. (2015): Institutional development for stakeholder participation in local water management: An analysis of two Swedish catchments. Land Use Policy 43, 217-227.

Gil Olcina, A. (2016): Acondicionamiento, rectificación y regulación del Segura. Modificación de lechos, cuenca y régimen fluvial. Publicacions Universitat d'Alacant, Alacant, 332 pp. 
Gómez-Baggethun, E., de Groot, R., Lomas, P.L. y Montes, C. (2010): The history of ecosystem services in economic theory and practice: from early notions to markets and payment schemes. Ecological Economics 69, 1209-1218.

Guilló, M. y Montaner, J.M. (2014): Entre acequias y azarbes: el legado del agua en el entorno de la Albufera de Elche como revulsivo para un territorio. En Sanchis-Ibor, C., Palau-Salvador, G., Mangue Alférez, I. y Martínez-Sanmartín, L.P. (ed.): Irrigation, Society, Landscape. Tribute to Thomas F. Glick. Universitat Politècnica de València, València, pp. 1187-1204.

Gupta, J., Akhmouch, A., Cosgrove, W., Hurwitz, Z., Maestu, J. y Ünver, U. (2013): Policymakers' reflections on water governance issues. Ecology and Society 18 (1), 35.

Gutzler, C., Helming, K., Balla, D., Dannowski, R., Deumlich, D., Glemnitz, M., Knierim, A., Mirschel, W., Nendel, C., Paul, C., Sieber, S., Stachow, U., Starick, A., Wieland, R., Wurbs, A. y Zander, P. (2015): Agricultural land use changes - a scenario-based sustainability impact assessment for Brandenburg, Germany. Ecological Indicators 48, 505-517.

Hernández, M. (2008): Balance de las políticas de desarrollo rural en la Comunidad Valenciana (19912006). Investigaciones Geográficas 45, 93-119.

Hileman, J., Hicks, P. y Jones, R. (2016): An alternative framework for analysing and managing conflicts in integrated water resources management (IWRM): Linking theory and practice. International Journal of Water Resources Development 32(5), 675-691.

Hoolohan, C., Larkin, A., Mclachlan, C., Falconer, R., Soutar, I., Suckling, J., Varga, L., Haltas, I., Druckman, A., Lumbroso, D., Scott, M., Gilmour, Ledbetter, R., Mcgrane, S., Mitchell, C., Yu, D. (2018): Engaging stakeholders in research to address water-energy-food (WEF) nexus challenges. Sustainability Science 13(5), 1415-1426.

Howlett, M., Rayner, J. y Tollefson, C. (2009): From government to governance in forest planning? Lessons from the case of the British Columbia Great Bear Rainforest initiative. Forest Policy and Economics 11(5-6), 383-391.

Huang, J., Tichit, M., Poulot, M., Darly, S., Li, S., Petit, C., Aubry, C. (2015): Comparative review of multifunctionality and ecosystem services in sustainable agriculture. Journal of Environmental Management 149, 138-147.

Jacobs, M.H. y Buijs, A.E. (2011): Understanding stakeholders' attitudes toward water management interventions: Role of place meanings. Water Resources Research 47(1), W01503.

Jafary, F. y Bradley, C. (2018): Groundwater irrigation management and the existing challenges from the farmers' perspective in Central Iran. Land 7(1), 15.

Kampen, J.K. (2019): Reflections on and test of the metrological properties of summated rating, Likert, and other scales based on sums of ordinal variables. Measurement 137, 428-434.

Karali, E., Brunner, B., Doherty, R., Hersperger, H. y Rounsevell, M. (2014): Identifying the factors that influence farmer participation in environmental management practices in Switzerland. $\mathrm{Hu}$ man Ecology 42, 951-963. 
Kharel, G., Joshi, O., Miller, R. y Zou, C. (2018): Perceptions of government and research experts' groups and their implications for watershed management in Oklahoma, USA. Environmental Management 62(6), 1048-1059.

Lang, D.J., Wiek, A., Bergmann, M., Stauffacher, M., Martens, P., Moll, P., Swilling, M. y Thomas, C.J. (2012): Transdisciplinary research in sustainability science: Practice, principles, and challenges. Sustainability Science 7(1), 25-43.

Levidow, L., Zaccaria, D., Maia, R., Vivas, E., Todorovic, M. y Scardigno, A. (2014): Improving water-efficient irrigation: prospects and difficulties of innovative practices. Agricultural Water Management 146, 84-94.

Lika, A., Galioto, F. y Viaggi, D. (2017): water authorities' pricing strategies to recover supply costs in the absence of water metering for irrigated agriculture. Sustainability 9(12), 2210.

Loft, L., Mann, C. y Hansjürgens, B. (2015): Challenges in ecosystem services governance: Multi-levels, multi-actors, multi-rationalities. Ecosystem Services 16, 150-157.

López-Gunn, E. (2009): Agua para todos: A new regionalist hydraulic paradigm in Spain. Water Alternatives 2(3), 370-394.

Lubell, M. y Edelenbos, J. (2013): Integrated water resources management: A comparative laboratory for water governance. International Journal of Water Governance 1, 177-196.

Marcantonio, R.A., Atari, S.Z. y Evans, T.P. (2018): Farmer perceptions of conflict related to water in Zambia. Sustainability 10(2): 313.

Martín Cantarino, M. (2010): Environmental conflicts and conflict management: some lessons from the WADI experience at El Hondo Nature Pak (South-Eastern Spain). En Scapini, F. y Ciampi, G. (eds.): Coastal water bodies: nature and culture conflicts in the Mediterranean. Springer, Dordrecht, pp. 61-78.

Martínez-Pérez, J.E., Belda-Antolí, A., Martín Cantarino, C., Seva-Román, E. (2009): Long-term (1883 to 2008) historical changes in agricultural landscape patterns in a traditional irrigated system of Alicante Province, Spain: the case of Lo Contador property (Carrizales of Elche). En Scapini, F., Boffa, J.M., Cassar, L.F., Conrad, E., Nardi, M. (eds.): Sustainable management of Mediterranean coastal fresh and transitional water bodies: a socio-economic and environmental analysis of changes and trends to enhance and sustain stakeholder benefits. Firenze University Press, Firenze, pp. 29-36.

Mollinga, P.P. (2008): Water, politics and development: Framing a political sociology of water resource management. Water Alternatives 1(1), 7-23.

Montgomery, J., Xu, W., Njornlund, H. y Edwards, J. (2016): A table for five: Stakeholder perceptions of water governance in Alberta. Agricultural Water Management 174, 11-21.

Morgan, S.L., Marsden, T., Miele, M. y Morley, A. (2010): Agricultural multifunctionality and farmers' entrepreneurial skills: A study of Tuscan and Welsh farmers. Journal of Rural Studies 26, 116-129.

Moore, M.L. (2013): Perspectives of complexity in water governance: Local experiences of global trends. Water Alternatives 6: 487-505. 
Munaretto, S., Siciliano, S. y Turvani, M.E. (2014): Integrating adaptive governance and participatory multicriteria methods: A framework for climate adaptation governance. Ecology and Society 19(2), 74.

Niño, N.S., Segrelles, J.A., Niño, I.N., Niño, J.E. (2015): Multifuncionalidad y turismo en el parque natural "El Hondo" de la Comunitat Valenciana. El Periplo Sustentable 29, 34-56.

Obermeister, N. (2017): From dichotomy to duality: Addressing interdisciplinary epistemological barriers to inclusive knowledge governance in global environmental assessments. Environmental Science and Policy 68, 80-86.

Page, G. y Bellotti, G. (2015): Farmers value on-farm ecosystem services as important, but what are the impediments to participation in PES schemes? Science of the Total Environment 515-516, 12-19.

Peng, J., Liu, Z., Liu, Y., Hu, X., Wang, A. (2015): Multifunctionality assessment of urban agriculture in Beijing City, China. Science of the Total Environment 537, 343-351.

Perramond, E.P. (2018): Water: Abundance, scarcity, and security in the Age of Humanity. Environmental History 23(3), 654-656.

Prager, K. (2015): Agri-environmental collaborative for landscape management in Europe. Current Opinion in Environmental Sustainability 12, 59-66.

Ricart, S. (2016): Entre la multifuncionalitat i la legitimitat social de l'agricultura: un estat de la qüestió. Documents d'Anàlisi Geogràfica 62(1),161-181.

Ricart, S. y Clarimont, S. (2016): Modelling the links between irrigation, ecosystem services and rural development in pursuit of social legitimacy: Results from a territorial analysis of the Neste System (Hautes-Pyrénées, France). Journal of Rural Studies 43, 1-12.

Ricart, S., Rico, A., Kirk, N., Bulöw, F., Ribas, A. y Pavón, D. (2018). How to improve governance in multifunctional irrigation systems? Balancing stakeholder engagement in hydrosocial territories. International Journal of Water Resources Development. In press. DOI: 10.1080/07900627.2018.1447911.

Rizzo, F. (2016): Approaching multifunctionality by a 'normative view': Finnish farmers' visions on contemporary agriculture. European Countryside 1, 16-32.

Ruiz, C. (2014): Valoración económica de los parques naturales del sur de la provincia de Alicante. Tesis doctoral. Universidad Miguel Hernández, Elche.

Rutz, C., Dwyer, J. y Schramek, J. (2013): More new wine in the same old bottles? The evolving nature of the CAP reform debate in Europe, and prospects and future. Sociologia Ruralis 54(3), 266-284.

Schulz, C., Martin-Ortega, J., Glenk, C. y Ioris, A. (2017): The value base of water governance: A multi-disciplinary perspective. Ecological Economics 131, 241-249.

Segrelles, J.A. (2013): Conservación ambiental y aprovechamiento agropecuario: El caso del Parque Natural Agrario "Los Carrizales" (Elche, Alicante). Boletín de la Asociación de Geógrafos Españoles 62, 295-316.

Seufert, V., Ramankutty, N. y Foley, J.A. (2012): Comparing the yields of organic and conventional agriculture. Nature 485, 229-234. 
Seuneke, P., Lans, T. y Wiskerke, J.S.C. (2013): Moving beyond entrepreneurial skills: key factors driving entrepreneurial learning in multifunctional agriculture. Journal of Rural Studies 32(2), 208219.

Silva, R. (2009): Agricultura, paisajes y patrimonio territorial. Los paisajes de la agricultura vistos como patrimonio. Boletín de la Asociación de Geógrafos Españoles 49, 309-334.

Sinclair, A.J., Kumnerdpet, W. y Moyer, J.M. (2013): Learning sustainable water practices through participatory irrigation management in Thailand. Natural Resources Forum 37(1), 55-66.

Smidt, S.J., Haacker, E.M.K., Kendall, A.D., Deines, J.M., Pei, L., Cotterman, K.A., et al. (2016): Complex water management in modern agriculture: Trends in the water-energy food nexus over the high plains aquifer. Science of the Total Environment 566-567, 988-1001.

Swinton, S.M., Lupi, F., Robertson, G.P. y Hamilton, S.K. (2007): Ecosystem services and agriculture: cultivating agricultural ecosystems for diverse benefits. Ecological Economics 64, 245-252.

Trapote, A., Roca, J.F., y Melgarejo, J. (2015): Azudes y acueductos del sistema de riego tradicional de la Vega Baja del Segura (Alicante, España). Investigaciones Geográficas 63, 143-160.

Varis, O., Enckell, K. y Keskinen, M. (2014): Integrated water resources management: Horizontal and vertical explorations and the 'water in all policies' approach. International Journal of Water Resources Development 30(3), 433-444.

When, U., Rusca, M., Evers, J. y Lanfranchi, V. (2015): Participation in flood risk management and the potential of citizen observatories: A governance analysis. Environmental Science $\mathcal{E}$ Policy 48, 225-236.

Wilson, G.A. (2009): The spatiality of multifunctional agriculture: a human geography perspective. Geoforum 40, 269-280.

Yao, L., Zhao, M. y Xu, T. (2017): China's water-saving irrigation management system: Policy, implementations and challenge. Sustainability 9 (12), 2339.

Cómo citar este artículo:

Ricart, S., Rico, A. M. (2019). Gobernanza y ecología política en la gestión del regadío multifuncional.

Aplicación al corredor agroecológico El Hondo-Los Carrizales (Alicante). Cuadernos de Geografía, 103, 25-46.

https://doi.org/10.7203/CGUV.103.16014

\section{(9) (1) $\Theta \Theta$}

Este obra está bajo una licencia de Creative Commons Reconocimiento-NoComercial-SinObraDerivada 4.0 Internacional. 
